\title{
NONTRIVIAL SOLUTIONS OF ASYMPTOTICALLY LINEAR SECOND ORDER INDEFINITE HAMILTONIAN SYSTEMS
}

\section{YINGYING CHEN and JINHANG XU}

Department of Mathematics

Nanjing Normal University

Nanjing, Jiangsu 210023

P. R. China

e-mail: chenyingying0620@163.com

xujinhang305@163.com

\begin{abstract}
Capietto, Dalbono and Portaluri proposed a class of strongly indefinite second order Hamiltonian systems with Dirichlet boundary conditions. We will investigate this system and obtain some new results.
\end{abstract}

\section{Introduction and Main Results}

Many famous mathematicians such as Ekeland [1]; Long [2, 3]; Mawhin and Willem [4]; and Rabinowitz [5] have investigated Hamiltonian systems. In [6, 7, 8, 9], some problems concerning Hamiltonian systems have also been investigated. In 2010, Capietto et al. [10] proved a multiplicity result for the class of strongly indefinite nonlinear second-order asymptotically linear systems with Dirichlet boundary conditions:

2010 Mathematics Subject Classification: 34A12, 34B15, 34L30, 49R50.

Keywords and phrases: strongly indefinite, Hamiltonian system, Dirichlet conditions.

Received May 11, 2015

(ㄷ) 2015 Scientific Advances Publishers 


$$
\begin{gathered}
J \ddot{x}+S(t, x(t)) x(t)=0, \\
x(0)=0=x(1),
\end{gathered}
$$

where $J=\left(\begin{array}{cc}I_{n-\nu} & 0 \\ 0 & -I_{\nu}\end{array}\right), S:[0,1] \times \mathbf{R}^{n} \rightarrow \mathcal{L}_{s}\left(\mathbf{R}^{n}\right)$ is continuous. We first introduce an index theory for the linear second order indefinite Hamiltonian system (1.2) and

$$
P \ddot{x}+B(t) x=0,
$$

where $P=\left(\begin{array}{cc}1 & 0 \\ 0 & -1\end{array}\right), B \in L^{\infty}\left([0,1], \mathcal{L}_{s}\left(\mathbf{R}^{2}\right)\right)$ in Section 2. Then, in Section 3, we investigate nontrivial solutions of the asymptotically linear second-order indefinite Hamiltonian system (1.2) and

$$
P \ddot{x}+V^{\prime}(t, x)=0,
$$

where $V \in C^{1}\left([0,1], \mathbf{R}^{2}\right)$.

In Section 4, we will recall some results from [11] concerning index theory for self-adjoint operator equations and multiple solutions for asymptotically linear operator equations, which are used in Sections 2-3. Our main results will be obtained by applying some associated results for operator equations from [11].

\section{Index Theory for Second Order Linear Indefinite Hamiltonian Systems}

In this chapter, we discuss the problem

$$
\begin{aligned}
& P \ddot{x}+B(t) x=0, \\
& x(0)=0=x(1),
\end{aligned}
$$

where $P=\left(\begin{array}{cc}1 & 0 \\ 0 & -1\end{array}\right), B \in L^{\infty}\left([0,1], \mathcal{L}_{s}\left(\mathbf{R}^{2}\right)\right)$. 
Define $\quad X=L^{2}\left([0,1], \mathbf{R}^{2}\right),(A x)(t)=-P \ddot{x}(t)$ with $D(A)=\left\{x \in H^{2}\right.$ $\left.\left([0,1] ; \mathbf{R}^{2}\right) \mid x(0)=0=x(1)\right\}$, then $A: D(A) \subset X \rightarrow X$.

We now prove $A$ is self-adjoint. If so, $\sigma_{r}(A)=\emptyset$. To this end, it suffices to prove

(i) $(A x, y)=(x, A y), \forall x, y \in D(A)$, and

(ii) $(A x, y)=(x, z), \forall x \in D(A) \Rightarrow y \in D(A)$, and $A y=z$.

In fact $\forall x, y \in D(A)$,

$$
\begin{aligned}
(A x, y) & =\int_{0}^{1}(-P \ddot{x}(t), y(t)) d t=\int_{0}^{1}-\ddot{x}_{1}(t) y_{1}(t) d t+\int_{0}^{1} \ddot{x}_{2}(t) y_{2}(t) d t \\
& =-\left.\dot{x}_{1}(t) y_{1}(t)\right|_{0} ^{1}+\int_{0}^{1} \dot{x}_{1}(t) \dot{y}_{1}(t) d t+\left.\dot{x}_{2}(t) y_{2}(t)\right|_{0} ^{1}-\int_{0}^{1} \dot{x}_{2}(t) \dot{y}_{2}(t) d t \\
& =\int_{0}^{1} \dot{x}_{1}(t) \dot{y}_{1}(t) d t-\int_{0}^{1} \dot{x}_{2}(t) \dot{y}_{2}(t) d t \\
& =\left.x_{1}(t) \dot{y}_{1}(t)\right|_{0} ^{1}-\int_{0}^{1} x_{1}(t) \ddot{y}_{1}(t) d t-\left.x_{2}(t) \dot{y}_{2}(t)\right|_{0} ^{1}+\int_{0}^{1} x_{2}(t) \ddot{y}_{2}(t) d t \\
& =-\int_{0}^{1} x_{1}(t) \ddot{y}_{1}(t) d t+\int_{0}^{1} x_{2}(t) \ddot{y}_{2}(t) d t \\
& =\int_{0}^{1}(x(t),-P \ddot{y}(t)) d t \\
& =(x, A y) .
\end{aligned}
$$

Thus (i) holds. To prove (ii), suppose

$$
\int_{0}^{1}(-P \ddot{x}(t), y(t)) d t=\int_{0}^{1}(x(t), z(t)) d t, \quad \forall x \in D(A) .
$$


Because $z \in L^{2}\left([0,1], \mathbf{R}^{2}\right)$, there exists

$$
w \in H_{0}^{2}\left([0,1] ; \mathbf{R}^{2}\right) \equiv D(A)
$$

such that $A w=z$. In fact, there exists

$$
x(t)=-t \int_{0}^{1} \int_{0}^{s} f(\tau) d \tau d s+\int_{0}^{t} \int_{0}^{s} f(\tau) d \tau d s=\int_{0}^{1} G(t, s) f(s) d s,
$$

with $G(t, s)=(t-1) s$ when $s \leq t$ and $G(t, s)=t(s-1)$ when $s>t$. $x(t) \quad$ is $\quad$ a solution of $\ddot{x}(t)=f(t), t \in(0,1), x(0)=0=x(1)$. Thus $w=-P \int_{0}^{1} G(t, s) z(s) d s$, and $(A x, y)=(x, A w)=(A x, w)$ so $(A x, y-w)=0$, $\forall x \in D(A)$, hence $y=w \in D(A)$.

Now, we want to prove $\sigma(A)=\sigma_{d}(A)$, and it suffices to prove

(i) $\sigma_{p}(A)=\left\{ \pm k^{2} \pi^{2}\right\}$;

(ii) $\operatorname{dim} \operatorname{ker}\left(A-\lambda I_{2}\right) \leq 1$ for $\lambda= \pm k^{2} \pi^{2}$;

(iii) $\sigma_{c}(A)=\emptyset$.

At first $\lambda \in \sigma_{p}(A) \Leftrightarrow \exists x \in D(A) \backslash\{0\}$ such that $\left(A-\lambda I_{2}\right) x=0$, equivalently, $-P \ddot{x}-\lambda x=0, x(0)=0=x(1)$.

$$
\begin{aligned}
& \text { By substituting } P=\left(\begin{array}{cc}
1 & 0 \\
0 & -1
\end{array}\right) \text {, we obtain } \\
& \ddot{x}_{1}+\lambda x_{1}=0, \quad \ddot{x}_{2}-\lambda x_{2}=0, \\
& x_{1}(0)=0=x_{1}(1), \quad x_{2}(0)=0=x_{2}(1) .
\end{aligned}
$$

So $\lambda \in\left\{ \pm k^{2} \pi^{2}\right\}$ for $k \in N^{*}$ and (i) holds. 
By definition, $\operatorname{ker}\left(A-\lambda I_{2}\right)=\left(\begin{array}{c}c \sin k \pi t \\ 0\end{array}\right)$ when $\lambda=k^{2} \pi^{2}, \quad$ and $\operatorname{ker}\left(A-\lambda I_{2}\right)=\left(\begin{array}{c}0 \\ c \sin k \pi t\end{array}\right)$ when $\lambda=-k^{2} \pi^{2}$, where $c$ is a constant, otherwise $\operatorname{ker}\left(A-\lambda I_{2}\right)=\{0\}$.

(iii) That $\sigma_{c}(A)=\emptyset$ is equivalent to the following: given $\lambda \in \mathbf{R} \backslash \sigma_{p}(A)$, $R\left(A-\lambda I_{2}\right)=X$. This is equivalent to $\forall f \in X,-P \ddot{x}-\lambda x=f$, $x(0)=0=x(1)$ has a unique solution. So, we have three possibilities:

Case 1. $\lambda=0$. In this case, $-P \ddot{x}=f(t), t \in(0,1), x(0)=0=x(1)$ has a unique solution: $x=-P \int_{0}^{1} G(t, s) f(s) d s$.

Case 2. $\lambda>0$. Set $\lambda=u^{2}, u>0$. Then, suppose the solution of $\ddot{x}+u^{2} x=f(t)$ is

$$
x(t)=c_{1}(t) \cos u t+c_{2}(t) \sin u t .
$$

Because

$$
\dot{x}(t)=-c_{1}(t) u \sin u t+c_{2}(t) u \cos u t+\dot{c}_{1}(t) \cos u t+\dot{c}_{2}(t) \sin u t .
$$

By setting

$$
\dot{c}_{1}(t) \cos u t+\dot{c}_{2}(t) \sin u t=0,
$$

and then

$$
\ddot{x}(t)=-u^{2} x(t)-\dot{c}_{1}(t) u \sin u t+\dot{c}_{2}(t) u \cos u t,
$$

$x(0)=0$ implies $c_{1}=0$, and because $x(1)=0$,

$$
0=-\frac{1}{u} \int_{0}^{1} f(\tau) \sin u \tau d \tau \cos u+\left(c_{2}+\frac{1}{u} \int_{0}^{1} f(\tau) \cos u \tau d \tau\right) \sin u
$$

Because $\lambda=u^{2}, \sin u \neq 0, c_{2}$ is determined uniquely, and hence $\ddot{x}+u^{2} x=f(t), x(0)=0=x(1)$ has a unique solution. 
Case 3. $\lambda<0$, let $\lambda=-u^{2}$, with $u>0$.

Suppose the solution of $\ddot{x}-u^{2} x=f(t)$ is

$$
x(t)=c_{1}(t) e^{-u t}+c_{2}(t) e^{u t} .
$$

Then

$$
\dot{x}(t)=-u c_{1}(t) e^{-u t}+u c_{2}(t) e^{u t}+\dot{c}_{1}(t) e^{-u t}+\dot{c}_{2}(t) e^{u t} .
$$

By setting

$$
\begin{gathered}
\dot{c}_{1}(t) e^{-u t}+\dot{c}_{2}(t) e^{u t}=0 . \\
\ddot{x}(t)=u^{2} x(t)-u \dot{c}_{1}(t) u^{-u t}+u \dot{c}_{2}(t) u^{u t} .
\end{gathered}
$$

Substituting into the equation $\ddot{x}-u^{2} x=f(t)$, we obtain

$$
-u \dot{c}_{1}(t) e^{-u t}+u \dot{c}_{2}(t) e^{u t}=f(t)
$$

Thus

$$
2 \dot{c}_{1}(t) e^{-u t}=-\frac{1}{u} f(t), \quad 2 \dot{c}_{2}(t) e^{u t}=\frac{1}{u} f(t),
$$

and

$$
\begin{aligned}
& c_{1}(t)=-\frac{1}{2 u}\left(\int_{0}^{t} f(\tau) e^{u \tau} d \tau+c_{1}\right), \\
& c_{2}(t)=\frac{1}{2 u}\left(\int_{0}^{t} f(\tau) e^{-u \tau} d \tau+c_{2}\right) .
\end{aligned}
$$

Finally,

$$
x(t)=-\frac{1}{2 u}\left(\int_{0}^{t} f(\tau) e^{u \tau} d \tau+c_{1}\right) e^{-u t}+\frac{1}{2 u}\left(\int_{0}^{t} f(\tau) e^{-u \tau} d \tau+c_{2}\right) e^{u t} .
$$


Because $x(0)=0=x(1), c_{1}$ and $c_{2}$ satisfy

$$
c_{1}-c_{2}=0, \quad\left(\int_{0}^{1} f(\tau) e^{-u \tau} d \tau+c_{2}\right) e^{u}-\left(\int_{0}^{1} f(\tau) e^{u \tau} d \tau+c_{1}\right) e^{-u}=0 .
$$

Then $c_{1}$ and $c_{2}$ are determined uniquely and it means that

$$
\ddot{x}-u^{2} x=f(t), \quad x(0)=0=x(1)
$$

has a unique solution.

Similar to Definition 4.1, we have the following definitions:

Definition 2.1. For any $\bar{B} \in L^{2}\left([0,1], \mathcal{L}_{s}\left(\mathbf{R}^{2}\right)\right)$, we define

$$
\nu_{P}(\bar{B}) \equiv \operatorname{dim} \operatorname{ker}(A-B)
$$

where $B \in \mathcal{L}_{s}(X)$ is defined by $(B x)(t)=\bar{B}(t) x(t)$.

Definition 2.2. For any $\bar{B}_{1}, \bar{B}_{2} \in L^{2}\left([0,1], \mathcal{L}_{s}\left(\mathbf{R}^{2}\right)\right)$ with $\bar{B}_{1}<\bar{B}_{2}$, we define

$$
I_{P}\left(\bar{B}_{1}, \bar{B}_{2}\right) \equiv \sum_{\lambda \in[0,1)} \nu_{P}\left(\left(\bar{B}_{1}+\lambda\left(\bar{B}_{2}-\bar{B}_{1}\right)\right)\right)
$$

and for any $\bar{B}_{1}, \bar{B}_{2} \in L^{2}\left([0,1], \mathcal{L}_{s}\left(\mathbf{R}^{2}\right)\right)$, we define

$$
I_{P}\left(\bar{B}_{1}, \bar{B}_{2}\right)=I_{P}\left(\bar{B}_{1}, c I_{2}\right)-I_{P}\left(\bar{B}_{2}, c I_{2}\right)
$$

for some constant $c$ satisfying $c I_{2}>\bar{B}_{1}, c I_{2}>\bar{B}_{2}$.

Here for any $\bar{B}_{1}, \bar{B}_{2} \in L^{2}\left([0,1], \mathcal{L}_{s}\left(\mathbf{R}^{2}\right)\right)$, we write $\bar{B}_{1} \leq \bar{B}_{2}$ if $\bar{B}_{1}(t) \leq \bar{B}_{2}(t)$ for a.e. $t \in[0,1]$; write $\quad \bar{B}_{1}<\bar{B}_{2} \quad$ if $\quad \bar{B}_{1} \leq \bar{B}_{2}$ and $\bar{B}_{1}(t)<\bar{B}_{2}(t)$ on a subset of $[0,1]$ with positive measure.

Definition 2.3. For any $\bar{B} \in L^{\infty}\left([0,1], \mathcal{L}_{s}\left(\mathbf{R}^{2}\right)\right)$, we define

$$
i_{P}(\bar{B})=I_{P}(0, B) .
$$


From Definitions 2.1-2.3, $\nu_{P}\left(c I_{2}\right)=0$ when $c \in \mathbf{R} \backslash\left\{ \pm k^{2} \pi^{2}\right\}(k \neq 0)$, $v_{P}\left(c I_{2}\right)=1$ when $c \in\left\{ \pm k^{2} \pi^{2}\right\} ; i_{P}\left(c I_{2}\right)=k$ when $|c| \in\left(k^{2} \pi^{2},(k+1)^{2} \pi^{2}\right]$, $i_{P}\left(c I_{2}\right)=0$ when $c \in\left(-\pi^{2}, \pi^{2}\right)$.

Proposition 2.4. For any $\bar{B}_{1}, \bar{B}_{2} \in L^{2}\left([0,1], \mathcal{L}_{s}\left(\mathbf{R}^{2}\right)\right)$. If $\bar{B}_{1} \leq \bar{B}_{2}$, then $i_{P}\left(\bar{B}_{1}\right) \leq i_{P}\left(\bar{B}_{2}\right), i_{P}\left(\bar{B}_{1}\right)+v_{P}\left(\bar{B}_{1}\right) \leq i_{P}\left(\bar{B}_{2}\right)+v_{P}\left(\bar{B}_{2}\right)$; if $\bar{B}_{1}<\bar{B}_{2}$, then $i_{P}\left(\bar{B}_{1}\right)+\nu_{P}\left(\bar{B}_{1}\right) \leq i_{P}\left(\bar{B}_{2}\right)$.

This proposition comes from [11, Proposition 5.1.2 (iii)].

\section{Nontrivial Solutions for Asymptotically Linear Second Order Indefinite Hamiltonian Systems}

In this chapter, we investigate the following problem:

$$
\begin{gathered}
P \ddot{x}+V^{\prime}(t, x)=0, \\
x(0)=0=x(1),
\end{gathered}
$$

where $V \in C^{1}\left([0,1] \times \mathbf{R}^{2 n}\right)$.

From Theorems 4.3-4.5, we have the following theorems:

Theorem 3.1. Assume that $V \in C^{1}\left([0,1] \times \mathbf{R}^{2}\right)$ satisfies

$\left(\mathrm{V}_{1}\right)$ there exist $\bar{B}_{1}, \bar{B}_{2} \in L^{\infty}\left([0,1], \mathcal{L}_{s}\left(\mathbf{R}^{2}\right)\right)$ with $i_{P}\left(\bar{B}_{1}\right)=i_{P}\left(\bar{B}_{2}\right)$, $\nu_{P}\left(\bar{B}_{2}\right)=0$ such that $V^{\prime}(t, x)=\bar{B}(t, x) x+h(t, x)$, where $\bar{B}_{1}(t) \leq \bar{B}(t, x)$ $\leq \bar{B}_{2}(t), \forall(t, x) \in C^{1}\left([0,1] \times \mathbf{R}^{2 n}\right)$ and $h(t, x)$ bounded, then (3.1)-(3.2) has one solution; if we assume

$\left(\mathrm{V}_{2}\right)$ there exist a symmetric $n \times n$ matrix $C(t, x)$, which is continuous with respect to $(t, x) \in[0,1] \times \mathbf{R}^{n}$ and $C_{1}, C_{2} \in L^{\infty}\left([0,1], \mathcal{L}_{s}\left(\mathbf{R}^{2}\right)\right)$ with $i_{P}\left(C_{1}\right)=i_{P}\left(C_{2}\right), \nu_{P}\left(C_{2}\right)=0$ such that $C_{1}(t) \leq C(t, x) \leq C_{2}(t)$, $\forall(t, x) \in[0,1] \times \mathbf{R}^{2}$ and $V^{\prime}(t, x)=C(t, x) x+o(|x|)$ as $|x| \rightarrow 0$. 
Then (3.1)-(3.2) has a nontrivial solution if $i_{P}\left(\bar{B}_{1}\right)-i_{P}\left(C_{1}\right)$ is odd.

Theorem 3.2. Assume that

$\left(\mathrm{V}_{3}\right) V \in C^{2}\left([0,1] \times \mathbf{R}^{2}\right)$ and $V^{\prime}(t, 0) \equiv 0 ;$

$\left(\mathrm{V}_{4}\right)$ there exist $\bar{B}_{1}, \bar{B}_{2} \in L^{\infty}\left([0,1], \mathcal{L}_{s}\left(\mathbf{R}^{2}\right)\right)$ satisfying $i_{P}\left(\bar{B}_{1}\right)=i_{P}\left(\bar{B}_{2}\right)$, $\nu_{P}\left(\bar{B}_{2}\right)=0$ and

$$
\bar{B}_{1}(t) \leq V^{\prime \prime}(t, x) \leq \bar{B}_{2}(t)
$$

$\forall(t, x)$ with $|x| \geq r>0$

$\left(\mathrm{V}_{5}\right)$ with $\bar{B}_{0} \equiv V^{\prime \prime}(t, 0)$, we have

$$
i\left(B_{1}\right) \notin\left[i_{P}\left(\bar{B}_{0}\right), i_{P}\left(\bar{B}_{0}\right)+\nu_{P}\left(\bar{B}_{0}\right)\right] .
$$

Then (3.1)-(3.2) has one nontrivial solution. Under the further assumption,

$$
\left(\mathrm{V}_{6}\right) \nu_{P}\left(\bar{B}_{0}\right)=0 \text { and }\left|i_{P}\left(\bar{B}_{1}\right)-i_{P}\left(\bar{B}_{0}\right)\right| \geq 2 \text {, (3.1)-(3.2) has two }
$$
nontrivial solutions.

Proof of Theorem 3.1. Define $X=L^{2}\left([0,1], \mathbf{R}^{2 n}\right),(A x)(t)=-P \ddot{x}(t)$, then $D(A)=\left\{x \in H^{2}\left([0,1], \mathbf{R}^{2}\right) \mid x(0)=0=x(1)\right\} \subset X . \quad$ As $\quad$ we have proved in Section 2, $A$ is an unbounded self-adjoint operator with $\sigma(A)=\sigma_{d}(A)$ is unbounded from both above and below and $Z \equiv D\left(|A|^{\frac{1}{2}}\right)$ $=H_{0}^{1}\left([0,1], \mathbf{R}^{2}\right)$ as we have proved in [12, Proposition 1.17]. Define

$$
\Phi(x)=\int_{0}^{1} V(t, x(t)) d t, \quad \forall x \in X
$$

By assumption $\left(\mathrm{V}_{1}\right), \Phi \in C^{1}(X)$ and

$$
\Phi^{\prime}(x) y=(\nabla \Phi(x), y)=\int_{0}^{1} V^{\prime}(t, x(t)) \cdot y(t) d t, \quad \forall x \in X .
$$


Set

$$
\begin{gathered}
(B(x) y)(t)=\bar{B}(t, x(t)) y(t), C(x)(t)=h(t, x(t)),\left(B_{i} x\right)(t)=\bar{B}_{i}(t) x(t), \\
i=1,2
\end{gathered}
$$

in view of Theorem 4.3, there exists one solution for (3.1)-(3.2); if we further assume $\left(\mathrm{V}_{2}\right)$, $\Phi$ defined as in (3.3) satisfies $\left(\Phi_{2}\right)$ of Theorem 4.3, then (3.1)-(3.2) has at least one nontrivial solution $i_{P}\left(\bar{B}_{1}\right)-i_{P}\left(C_{1}\right)$ is odd.

Proof of Theorem 3.2. The following trick comes from [11]. Note that

$$
V^{\prime}(t, x)=\int_{0}^{1} V^{\prime \prime}(t, \theta x) d \theta x+V^{\prime}(t, 0)
$$

Define

$$
B(t, x)=\int_{0}^{1} V^{\prime \prime}(t, \theta x) d \theta, \quad|x| \geq r / \delta
$$

and otherwise

$$
B(t, x)=B_{1}(t)
$$

When $\|x\| \geq r / \delta$

$$
\int_{0}^{1} V^{\prime \prime}(t, \theta x) d \theta=\int_{\delta}^{1}+\int_{0}^{\delta} V^{\prime \prime}(t, \theta x) d \theta=I_{1}+I_{2},
$$

then, in view of $\left(\mathrm{V}_{3}\right)$

$$
(1-\delta) B_{1}(t) \leq I_{1} \leq B_{2}(t)(1-\delta) .
$$

There exists $M>0$ such that

$$
-M \leq V^{\prime \prime}(t, x) \leq M,
$$


for all $(t, x) \in[0,1] \times \mathbf{R}^{n}$. Thus

$$
-M \delta \leq I_{2} \leq M \delta
$$

and if $B_{2}(t) \leq M$,

$$
I_{1}+I_{2} \leq B_{2}(t)+M \delta-B_{2}(t) \delta \leq B_{2}(t)+2 \delta M ;
$$

and at the same time $B_{1}(t) \leq B_{2}(t) \leq M$, then

$$
I_{1}+I_{2} \geq B_{1}(t)-\delta B_{1}(t)-\delta M \geq B_{1}(t)-2 \delta M .
$$

Because $i\left(B_{1}\right)=i\left(B_{2}\right)$ and $\nu\left(B_{1}\right)=\nu\left(B_{2}\right)=0$, there exists $\epsilon>0$ such that

$$
i\left(B_{1}-\epsilon\right)=i\left(B_{1}\right)=i\left(B_{2}\right)=i\left(B_{2}+\epsilon\right),
$$

and

$$
\nu\left(B_{1}-\epsilon\right)=0=\nu\left(B_{2}+\epsilon\right) .
$$

Choose $M>0$ such that

$$
-M \leq B_{1}(t) \leq B_{2}(t) \leq M,
$$

and $\delta>0$ such that

$$
2 \delta M<\epsilon .
$$

Then

$$
B_{1}(t)-\epsilon \leq B(t, x) \leq B_{2}(t)+\epsilon
$$

and

$$
h(t, x)=V^{\prime}(t, x)-B(t, x) x
$$

is bounded. Theorem 4.4 applies and the results hold.

\section{Corollary 3.3. Assume that}

$$
\left((k-1)^{2} \pi^{2}+\epsilon\right) I_{n} \leq V^{\prime \prime}(t, x) \leq\left(k^{2} \pi^{2}-\epsilon\right) I_{n}, \quad \forall(t, x),
$$

where $\epsilon>0$ is small and $k \in \mathbf{N}$. Then (3.1)-(3.2) has at least one solution. 
Proof. Let $B_{1}(t):=(k-1)^{2} \pi^{2} I_{n}, B_{2}(t):=\left(k^{2} \pi^{2}-\frac{\epsilon}{2}\right) I_{n}$, then $N(t, x):=V(t, x)-\frac{1}{2}\left(B_{1}(t) x, x\right)$ is convex with respect to $x$, since $N^{\prime \prime}(t, x) \geq \epsilon I_{2 n}$, and

$$
\begin{aligned}
V(t, x) & =\int_{0}^{1} d s\left(\int_{0}^{1} V^{\prime \prime}(t, \tau s x) x s d \tau, x\right)+\left(V^{\prime}(t, \theta), x\right)+V(t, \theta) \\
& \leq \frac{1}{2}\left(k^{2} \pi^{2}-\epsilon\right)|x|^{2}+\left(V^{\prime}(t, \theta), x\right)+V(t, \theta) \\
& \leq \frac{1}{2} b|x|^{2}+c,
\end{aligned}
$$

where $b:=k^{2} \pi^{2}-\frac{\epsilon}{2}, c>0$ is a constant. Since $i_{P}\left(b I_{n}\right)=i_{P}\left((k-1)^{2} \pi^{2} I_{n}\right)$ $+\nu_{P}\left((k-1)^{2} \pi^{2} I_{n}\right),(3.1)-(3.2)$ has a solution in view of Theorem 3.2.

In the end of this chapter as in Dong [11], we have the following result:

Theorem 3.4. Assume that V satisfies

$\left(\mathrm{V}_{7}\right) V \in C^{2}\left([0,1] \times \mathbf{R}^{2 n}\right)$ and there exist $B_{1}, B_{2} \in L^{\infty}\left([0,1], \mathcal{L}_{s}\left(\mathbf{R}^{2 n}\right)\right)$ with $i_{P}\left(B_{1}\right)=i_{P}\left(B_{2}\right), \nu_{P}\left(B_{2}\right)=0$ such that

$$
B_{1}(t) \leq V^{\prime \prime}(t, x) \leq B_{2}(t)
$$

$\forall(t, x) \in[0,1] \times \mathbf{R}^{2 n}$ with $|x| \geq r>0$.

$\left(\mathrm{V}_{8}\right) V^{\prime}(t, 0) \equiv 0$ and $\nu_{P}\left(B_{0}\right)=0$, where we set $B_{0}(t) \equiv V^{\prime \prime}(t, 0)$.

$\left(\mathrm{V}_{9}\right) V(t,-x)=V(t, x)$ for all $(t, x) \in[0,1] \times \mathbf{R}^{n}$,

then (3.1)-(3.2) has $\left|i_{P}\left(B_{0}\right)-i_{P}\left(B_{1}\right)\right|$ distinct pairs of solutions. 
Proof. From (3.4) and $\left(\mathrm{V}_{8}\right)$,

$$
\Phi^{\prime}(x) y=\int_{0}^{1} V^{\prime}(t, x) y d t=\int_{0}^{1} \int_{0}^{1} V^{\prime \prime}(t, \theta x) x y d \theta d t
$$

thus $\left(\Phi_{1}\right)$ is satisfied in view of $\left(\mathrm{V}_{7}\right)$.

And $\left(\Phi^{\prime \prime}(x) y, z\right)=\int_{0}^{1}\left(V^{\prime \prime}(t, x) y, z\right) d t$, with $V^{\prime \prime}(t, 0)=B_{0}(t)$ and $\nu_{P}\left(B_{0}\right)=0$, $\left(\Phi_{4}\right)$ is satisfied, thus Theorem 4.5 yields $\left|i_{P}\left(B_{0}\right)-i_{P}\left(B_{1}\right)\right|$ distinct pairs of solutions for (3.1)-(3.2).

\section{Appendix}

In this section, we will recall some results from [11] concerning index theory for self-adjoint operator equations and multiple solutions of asymptotically linear operator equations, which are used in Sections 2-3.

Let $X$ be a real separable infinite dimensional Hilbert space with inner product $(\cdot, \cdot)$ and norm $\|\cdot\|$. Let $A: D(A) \subset X \rightarrow X$ be an unbounded linear self-adjoint operator with domain $D(A)$ satisfying $\sigma(A)=\sigma_{d}(A)$ and which is unbounded from both above and below. In [11], the authors establish an index theory for the equation

$$
A x-B x=0,
$$

for any $B \in \mathcal{L}_{s}(X)$.

Definition 4.1. (i) For any $B \in \mathcal{L}_{s}(X)$, we define

$$
\nu_{A}(B)=\operatorname{dim} \operatorname{ker}(A-B),
$$

$\nu_{A}(B)$ is called the nullity of $B$.

(ii) For any $B_{1}, B_{2} \in \mathcal{L}_{s}(X)$ with $B_{1}<B_{2}$, we define

$$
I_{A}\left(B_{1}, B_{2}\right)=\sum_{\lambda \in[0,1)} v_{A}\left((1-\lambda) B_{1}+\lambda B_{2}\right)
$$


and for any $B_{1}, B_{2} \in \mathcal{L}_{s}(X)$, we define

$$
I_{A}\left(B_{1}, B_{2}\right)=I_{A}\left(B_{1}, k I\right)-I_{A}\left(B_{2}, k I\right),
$$

where $I: X \rightarrow X$ is the identity map and $k I>B_{1}, k I>B_{2}$ for some real number $k>0$.

(iii) For any $B \in \mathcal{L}_{s}(X)$, we define

$$
i_{A}(B)=i_{A}\left(B_{0}\right)+i_{A}\left(B_{0}, B\right)
$$

where $B_{0} \in \mathcal{L}_{s}(X)$ is fixed and $i_{A}\left(B_{0}\right)$ is a prescribed integer.

Here for any $B_{1}, B_{2} \in \mathcal{L}_{s}(X)$, we write $B_{1} \leq B_{2}$ with respect to $X_{1}$ (a linear subspace of $X$ ) if and only if $\left(B_{1} x, x\right) \leq\left(B_{2} x, x\right)$ for any $x \in X_{1}$; we write $B_{1}<B_{2}$ with respect to $X_{1}$ if and only if $\left(B_{1} x, x\right)<\left(B_{2} x, x\right)$ for any $x \in X_{1} \backslash\{0\}$. If $X_{1}=X$, we just write $B_{1} \leq B_{2}$ or $B_{1}<B_{2}$. As in [11], we call $I_{A}\left(B_{1}, B_{2}\right)$ the relative Morse index between $B_{1}$ and $B_{2}$, we call $i_{A}(B)$ the index of $B$ and $i_{A}\left(B_{0}\right)$ is called an initial index. Generally, the initial index can be any prescribed integer and the index $i_{A}(B)$ also depends on $B_{0}$ and the initial index. However, for a concrete operator $A$ in applications, we will choose special $B_{0}$ and $i_{A}\left(B_{0}\right)$ so that $i_{A}(B)$ looks like natural as much as possible.

The index and nullity established above have the following monotone property.

Proposition 4.2. For any $B_{1}, B_{2} \in L^{\infty}\left([0,1], \mathcal{L}_{s}\left(\mathbf{R}^{2 n}\right)\right)$, if $B_{1} \leq B_{2}$, then $i_{A}\left(B_{1}\right) \leq i_{A}\left(B_{2}\right)$ and $i_{A}\left(B_{1}\right)+v_{A}\left(B_{1}\right) \leq i_{A}\left(B_{2}\right)+v_{A}\left(B_{2}\right)$; if $B_{1}<B_{2}$, then $i_{A}\left(B_{1}\right)+v_{A}\left(B_{1}\right) \leq i_{A}\left(B_{2}\right)$.

Consider the following operator equation:

$$
A x-\nabla \Phi(x)=0
$$

where $\nabla \Phi: Z \equiv D\left(|A|^{\frac{1}{2}}\right) \rightarrow X$ satisfying $(\nabla \Phi(x), y)=\Phi^{\prime}(x) y, \forall x, y \in Z$ 
Theorem 4.3 [11, Theorem 8.4.1]. Assume that $\Phi \in C^{1}(Z, \mathbf{R})$ satisfies

$\left(\Phi_{1}\right)$ There exist $B: X \rightarrow \mathcal{L}_{s}(X), B_{1}, B_{2} \in \mathcal{L}_{s}(X)$ with $i_{A}\left(B_{1}\right)=i_{A}\left(B_{2}\right)$, $\nu_{A}\left(B_{2}\right)=0$ such that $B_{1} \leq B(x) \leq B_{2} \quad$ and $\nabla \Phi(x)-B(x) x=C(x)$, $\|C(x)\| \leq M$ for all $x \in Z$ and some $M>0$, then (4.6) has at least one solution, if we further assume that

$\left(\Phi_{2}\right)$ there exist $B_{0}: X \rightarrow \mathcal{L}_{s}(X)$ and $B_{01}, B_{02} \in \mathcal{L}_{s}(X)$ with $i_{A}\left(B_{01}\right)=i_{A}\left(B_{02}\right), v_{A}\left(B_{02}\right)=0$ and some $r>0$ such that $B_{01} \leq B_{0}(x) \leq B_{02}$, $\nabla \Phi(x)=B_{0}(x)$ for all $x \in D(A)$ with $\|x\| \leq r$.

Then (4.6) has one nontrivial solution provided $i_{A}\left(B_{1}\right)-i_{A}\left(B_{01}\right)$ is odd.

Theorem 4.4. Assume that

(i) $\Phi \in C^{2}(Z)$ with $Z:=D\left(|A|^{\frac{1}{2}}\right), \Phi^{\prime}(0)=0, \Phi^{\prime \prime}(x)$ exists and is bounded for $x \in X$;

(ii) there exist $B_{1}, B_{2} \in \mathcal{L}_{s}(X)$ with $i_{A}\left(B_{1}\right)=i_{A}\left(B_{2}\right), \nu_{A}\left(B_{2}\right)=0$ and $B: X \rightarrow \mathcal{L}_{s}(X), C: X \rightarrow X$ such that

$$
\begin{aligned}
& \Phi^{\prime}(x)=B(x) x+C(x) \text { for any } x, \\
& B_{1} \leq B(x) \leq B_{2}, C(x) \text { is bounded }
\end{aligned}
$$

(iii) with $B_{0}:=\Phi^{\prime \prime}(0)$, we have

$$
i_{A}\left(B_{1}\right) \notin\left[i_{A}\left(B_{0}\right), i_{A}\left(B_{0}\right)+v_{A}\left(B_{0}\right)\right] .
$$

Then (4.2) has a nontrivial solution $x=x_{0}$.

Under the further assumption that

(iv) $\nu_{A}\left(B_{0}\right)=0$ and $\left|i_{A}\left(B_{1}\right)-i_{A}\left(B_{0}\right)\right| \geq v_{A}\left(\Phi^{\prime \prime}\left(x_{0}\right)\right)$, (4.2) has two nontrivial solutions. 
Theorem 4.5 [11, Theorem 8.5.1]. Assume that $\Phi \in C^{1}\left(Z, \mathbf{R}^{2}\right)$ satisfies $\left(\Phi_{1}\right)$ and

$\left(\Phi_{3}\right) \Phi(-x)=\Phi(x), \forall x \in Z$,

$\left(\Phi_{4}\right) \Phi \in C^{2}(Z)$ and there exist $\widetilde{B}: Z \rightarrow \mathcal{L}_{s}(X)$ and $M>0$ with $|\widetilde{B}(x)| \leq M$ such that

$$
\Phi^{\prime \prime}(x)(u, v)=(\widetilde{B}(x) u, v), \quad \forall x, u, v \in Z,
$$

and let $B_{0} \equiv \widetilde{B}(0)$ with $\nu_{A}\left(B_{0}\right)=0$.

Then (4.6) has $\left|i_{A}\left(B_{0}\right)-i_{A}\left(B_{1}\right)\right|$ distinct pairs of nontrivial solutions.

\section{References}

[1] I. Ekeland, Convexity Methods in Hamiltonian Mechanics, Berlin, Springer, 1990.

[2] Y. Long, Index Theory and it's Applications in Hamiltonian Systems (in Chinese), Academic Press, 1993.

[3] Y. Long, Index Theory for Symplectic Paths with Applications, Progress in Mathematics, No. 207, Birkhauser, Basel, 2002.

[4] J. Mawhin and M. Willem, Critical Point Theory and Hamiltonian Systems, Springer, Berlin, 1998.

[5] P. Rabinowitz, Minimax methods in critical point theory with applications to differential equations, C. B. M. 65 (1986).

[6] A. Du, Morse Index Theory and Existence of Asymptotically Linear 2p-order Hamiltonian Systems, Nanjing Normal University, 2008.

[7] I. Ekeland, N. Ghoussoub and H. Terani, Multiple solutions for a classical problem in the calculus of varations, J. Differential Equations 131 (1996), 299-343.

[8] Z. Wang, Multiple solutions for indefinite functionals and applications to asymptotically linear problem, Acta Math. Sinica (N. S.) 5 (1989), 101-113.

[9] J. Yue, Index Theory and Existence of Asymptotically Linear Second Order Ordinary Differential Systems, Nanjing Normal University, 2008. 
[10] A. Capietto, F. Dalbono and A. Portaluri, A multiplicity result for a class of strongly indefinite asymptotically linear second order systems, Nonlinear Anal. 72 (2010), 2874-2890.

[11] Y. Dong, Index Theory for Hamiltonian Systems and Multiple Solution Problems, Science Press, Beijing, 2015.

[12] Y. Chen, Y. Dong and Y. Shan, Existence of solutions for sub-linear or super-linear operator equations, Science China Mathematics, Online preprint. 